科 学 通 报

\title{
小鼠脑内钙调神经磷酸酶内源底物的研究 *
}

\author{
孟祥兵 ** 魏 群 \\ (北京师范大学分子生物学及生物化学研究室, 北京 100875)
}

\section{关键词铝调神经磷酸醇、脑、内源底物}

钻调神经磷酸酶是哺乳动物脑内含量极丰富的唯一依赖 $\mathrm{Ca}^{2+}$ 及钙调素的磷蛋白磷酸 酶. 该酶 (calcineurin, $\mathrm{CaN}$ ) 在脑外组织如精子细胞、淋巴细胞及肌肉组织中也有分布, 但含 量远远低于脑 ${ }^{[1]}$. $\mathrm{CaN}$ 由 $18 \mathrm{kD} \mathrm{Ca}^{2+}$ 结合的调节亚基和 $61 \mathrm{kD}$ 钻调素结合的催化亚基组成. 除可与 $\mathrm{Ca}^{2+}$ 结合外, 该酶还可与 $\mathrm{Mn}^{2+}, \mathrm{Ni}^{2+}, \mathrm{Co}^{2+}$ 等金属离子结合而影响其活性. $\mathrm{CaN}$ 催化 亚基已有至少 5 种 $\mathrm{cDNA}$ 同型物分别从大鼠、小鼠和人基因库中调出. 这些基因型是从分别 位于人第 4 号和第 10 号染色体上的两类基因衍变而来的 ${ }^{[2]}$. 体外实验表明, $\mathrm{CaN}$ 与其它磷蛋 白磷酸酶相比, 底物范围较窄, 已知的体内底物更少. 已有证据表明 $\mathrm{CaN}$ 在精子鞭毛运动、钲 转运调节、细胞中环腺苷酸效应弱化等过程中起作用 ${ }^{[1]}$. 近年来又发现 $\mathrm{CaN}$ 在 $\mathrm{T}$ 细胞免疫抑 制中发挥重要作用 ${ }^{[3]}$. 免疫抑制剂如 FK506 与其胞内受体 FKBP 结合, 通过抑制 CaN 活力, 诘少调节白细胞介素 2(IL-2) 等细胞因子基因表达的转录因子的脱磷酸化, 从而抑制免疫细胞 因子的表达. 免疫组化分析 FKBP 在脑内也有大量分布, 而且在各脑区的分布与 CaN 相 同, 但其生理意义还不清楚. $\mathrm{CaN}$ 在含量丰富的神经组织中的生理功能目前所知很少, 本实 验试图通过对 $\mathrm{CaN}$ 脑内内源底物的研究为 $\mathrm{CaN}$ 在脑内生理功能的阐明提供线索.

\section{1 材 料 与 方 法}

钙调素、牛脑钙调神经磷酸酶及其多克隆抗体由本室纯化和制备. 实验用昆明种小鼠购 自北京医科大学实验动物部, 体重为 $18-22 \mathrm{~g}$ 雄性小鼠. 环腺苷酸 (cAMP)、磷酯酰丝氨酸 (PS)、PMA(phorbol-12-myristate 13-acetate)、去污剂CHAPS(3-\{(3-cholamidopropyl)-dimethylammonio]-1-proparesulfonate)、蛋白酶抑制剂 PMSF(phenylmethylsulfonyl fluoride) 为 Sigma 公司产品, 丙烯酰胺、甲叉双丙烯酰胺为 Bio-Rad 公司产品. 载体两性电解质为瑞典 LKB 公 司产品. $\gamma-{ }^{32}$ P-ATP 为北京福瑞公司产品. 其它为国产分析纯试剂.

\section{1 钙调素、铝调神经磷酸酶及其多克隆抗体的纯化及制备, $\mathrm{CaN}$ 抗体特异性、亲和力测定}

- 见文献[4,5].

\section{2 小鼠脑蛋白粗提液制备}

小鼠脑按 1:3加人匀浆液(20mmol/L Tris-HCl(pH7.4)-2mmol/L疏基乙醇-3mmol/L EGTA-

1993-08-23 收稿, 199401-06 收修改稿.

*国家自然科学基金资助项目.

** 现在地址: 军事医学科学院放射医学研究所四室, 北京 100850 . 
$0.1 \mathrm{mmol} / \mathrm{L}$ PMSF- $0.3 \%$ Triton X-100) 玻璃匀浆器 $4^{\circ} \mathrm{C}$ 匀浆, 超声破细胞膜. 然后在 $4^{\circ} \mathrm{C}$ 水 浴中放置 $20 \mathrm{~min}$ 以溶脱膜蛋白, $4^{\circ} \mathrm{C} 10000 \mathrm{r} / \mathrm{min}$ 离心 $20 \mathrm{~min}$. 上清液经玻璃棉脱脂即得小鼠 脑蛋白粗提液.

\section{$1.3 \mathrm{CaN}$ 内源底物测定}

方法, 参考 $\mathrm{Tash}^{[6]}$ 方法并加以改进. 小鼠脑蛋白粗提液中加人适量抗体, $4^{\circ} \mathrm{C}$ 水浴保温过 夜, 以封闭内源 $\mathrm{CaN}$ 活力作为加抗体组蛋白粗提液. 同时以加人与所加抗体等体积匀浆液的 小鼠脑蛋白粗提液作为对照组蛋白粗提液, $4^{\circ} \mathrm{C}$ 保温同样时间. 然后取加抗体组与对照组脑 蛋白粗提液分别与等体积磷酸化反应缓冲液混合, $30^{\circ} \mathrm{C}$ 进行 $5 \mathrm{~min}$ 体外磷酸化反应, 将脑内磷 蛋白标记放射性同位素 ${ }^{32} \mathrm{P}$. 由于加抗体组内 $\mathrm{CaN}$ 没有活力, 对照组内. $\mathrm{CaN}$ 有活力, 故在相 同活力激酶催化下, 加抗体组 $\mathrm{CaN}$ 内源底物标记 ${ }^{32} \mathrm{P}$ 比对照组强, 电泳后自显影照片中对应的 光密度就比对照组大. 标记 ${ }^{32} \mathrm{P}$ 的磷蛋白经 SDS- 聚丙烯酰胺凝胶电泳或双向电泳分离后 ${ }^{[7}$, 放射自显影, 比较加抗体组与对照组相应蛋白带曝光强弱就可鉴定 $\mathrm{CaN}$ 内源底物. 磷酸化反应 缓冲液为 $80 \mathrm{mmol} / \mathrm{L}$ Tris- $\mathrm{HCl}\left(\mathrm{pH} 7.4\right.$ ) $-20 \mathrm{mmol} / \mathrm{L} \mathrm{MgSO}_{4}-0.4 \mathrm{mmol} / \mathrm{L}$ EDTA- $10 \mathrm{mmol} / \mathrm{L} \beta$-颈基 乙醇 $-20 \mu \mathrm{mol} / \mathrm{L}$ ATP-3.0 $\mu \mathrm{mol} / \mathrm{L}$ PMA- $1 \mathrm{mmol} / \mathrm{L}$ PS- $20 \mu \mathrm{mol} / \mathrm{L} \mathrm{cAMP}-6.0 \mathrm{mmol} / \mathrm{L} \mathrm{CaCl}_{2}-40 \mu \mathrm{g} / \mathrm{ml}$ 锭调素- $6 \mu \mathrm{Ci} \gamma-{ }^{32} \mathrm{P}$-ATP.

\section{2 结 果与 讨 论}

采用蛋白印迹法检测小鼠脑内蛋白粗提液中的 $\mathrm{CaN}$, 由图 1 看到所用抗体可特异地识别 $\mathrm{CaN} 61$ 和 $18 \mathrm{kD}$ 两亚基.

利用高亲和力的抗 $\mathrm{CaN}$ 多克隆抗体, 按照材料与方法中 1.3 研究了小鼠脑内 $\mathrm{CaN}$ 内源底 物. 图 2(A) 为体外磷酸化标记 ${ }^{32} \mathrm{P}$ 的加抗体组 (b) 与对照组 (a) 小鼠脑磷蛋白经 $7.5-15 \%$

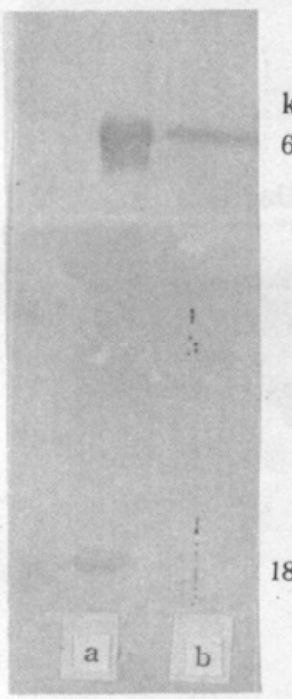

图 1 蛋白印迹法分析小鼠腹水 多克隆抗体特异性 $\mathrm{a}$ 为从牛脑中纯化的 $\mathrm{CaN}$ (上样量为 $15 \mu \mathrm{g}$ 蛋白), $\mathrm{b}$ 为小鼠脑蛋白粗提液 (上样量为 $50 \mu \mathrm{g}$ 蛋白)

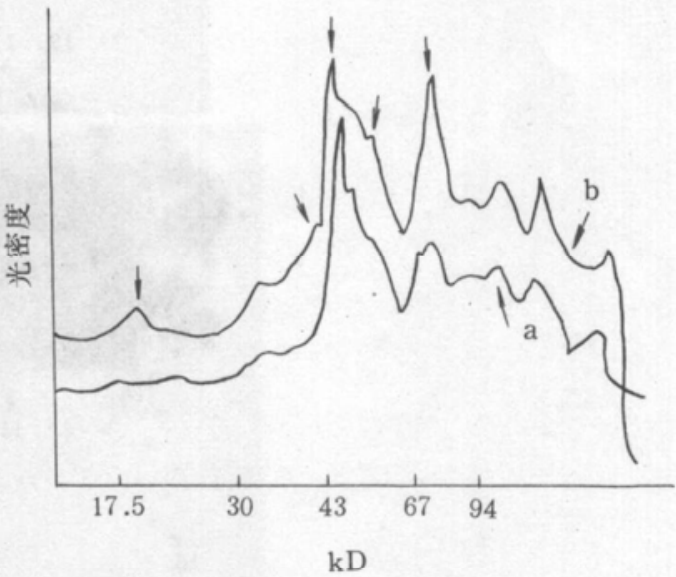

(B)

(A) 对照组 (a) 与加抗体组 (b) 小鼠脑蛋白体外磷酸化标记 ${ }^{32} \mathrm{P}$, 经 7.5-15\% SDS-PAGE 分离的放射自显影图; (B) 为相应的光密度扫描图 
SDS- 聚丙烯酰胺凝胶电泳分离后的放射自显影图, 图 2(B) 为相应的光密度扫描图. 由图 2 可 见, 有 5 条磷蛋白带光密度在加抗体组与对照组间有明显差异, 这就是 5 种 $\mathrm{CaN}$ 内源底物, 对 应的分子量是 $84,59,50,42$ 和 $17 \mathrm{kD}$ (见图 2(A) 中箭头所示). 由于测定 $\mathrm{CaN}$ 内源底物时, ${ }^{32} \mathrm{P}$ 标记 $\mathrm{CaN}$ 内源底物的同时, 其它脑内磷蛋白也标记了 ${ }^{32} \mathrm{P}$, 故电泳自显影照片有许多蛋白带, 某些 $\mathrm{CaN}$ 内源底物会被掩盖掉而无法鉴定. 另外, 只根据分子量很难判断某一带型为何种蛋 白. 所以我们进一步用高分辨率的双向电泳分离蛋白, 然后经放射自显影鉴定 $\mathrm{CaN}$ 内源底 物.

图 3 为双向电泳分析 $\mathrm{CaN}$ 内源底物图. 由图 3(a) 与 (b) 比较可见蛋白 1, 2, 3, 5, 6, 7, $10,12 \mathrm{a}, 12 \mathrm{~b}, 12 \mathrm{c}$ 和 13 为 $\mathrm{CaN}$ 内源底物. 图 3(c) 和 (d) 为过度曝光的自显影图, 由图可见标记 ${ }^{32} \mathrm{P}$ 较弱的 $\mathrm{CaN}$ 内源底物, 蛋白 $4,8,9,11$. 从图 3 中可检测到 15 种蛋白其磷酸化水平受 $\mathrm{CaN}$ 调节, 即检测到 15 种 $\mathrm{CaN}$ 内源底物. 经过与文献报道的鼠脑磷蛋白双向电泳放射自显 影图谱比较 ${ }^{[8-10]}$, 我们对较突出的 $\mathrm{CaN}$ 内源底物做了初步推断. 蛋白 1 为富含于脑的 PKC 内源底物 $87 \mathrm{~K}$ 蛋白. 蛋白 2 是神经系统特有的 B-50 蛋白 (又称 GAP-43 Neuromodulin). 该 蛋白既是 PKC 内源底物, 又可与钙调素结合, 是当前研究很活跃的一种蛋白. 蛋白 $12 \mathrm{a}, 12 \mathrm{~b}$, $12 \mathrm{c}$ 可能是一种蛋白的三种磷酸化形式, 可能是与第二信使传递关系密切的 Stathmin. 其它

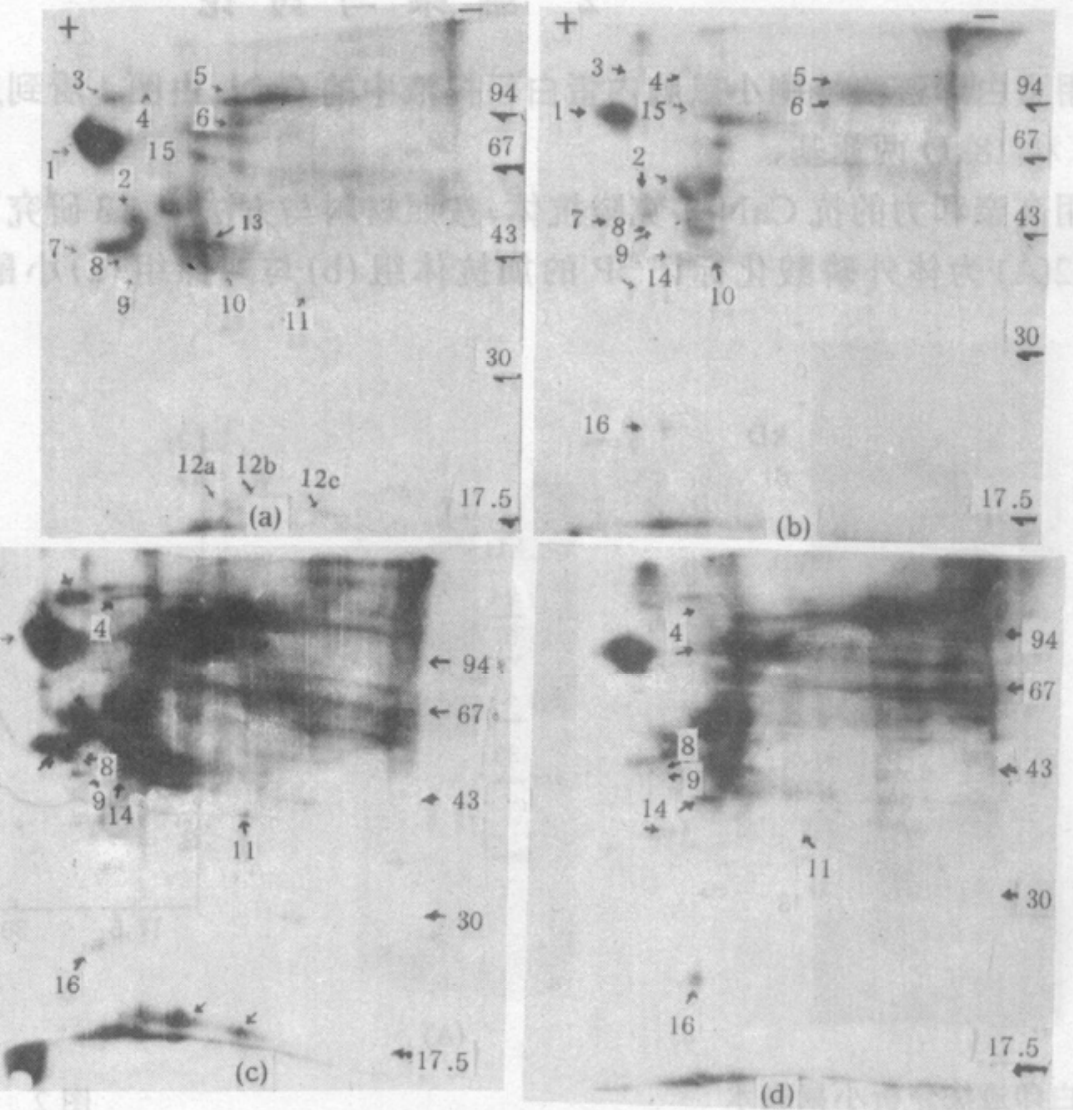

图 3 双向电泳分析 $\mathrm{CaN}$ 内源底物

加抗体组 ( (a), (c)) 与对照组 ((b), (d)) 小鼠脑蛋白体外磷酸化标记 ${ }^{32} \mathrm{P}$, 经双向电泳分离的放射自显影图.

(a), (b) 为正常曝光组 (自显影 4 天), (c), (d) 为过度曝光组 (自显影 10 天) 
内源底物还有待确证. 由图 3 还可看到蛋白 $14,15,16$ 在加抗体组标记 ${ }^{32} \mathrm{P}$ 比对照组弱, 原因 尚不清楚, Tash ${ }^{[6]}$ 等在研究精子中 $\mathrm{CaN}$ 内源底物时也观察到此现象.

本文报道了 15 种小鼠脑内 $\mathrm{CaN}$ 内源底物,并对其中较突出的几个根据其在双向电泳自 显影图谱上的位置做了初步推断, 为深人研究 $\mathrm{CaN}$ 脑内内源底物及 $\mathrm{CaN}$ 在神经系统的作用 提供了新的线索. 但我们对报道的内源底物所做的推断是初步的, 最后确证这 15 种内源底物 是何种蛋白尚需大量的证据. 进一步确证这些 $\mathrm{CaN}$ 内源底物将有助于阐明 $\mathrm{CaN}$ 在神经系统的 生理功能.

\section{参 考文献}

[1] Klee, C. B. et al., Molecular Aspects of Cellular Regulation, Amsterdam, New York, Oxford, 1988, $225-286$.

[2] Girl, P. R. et al., Biochem. Biophys Res. Commun., 1991, 181:252-258.

[ 3] Neil, A. C. et al. Nature, 1992, 357:695-697.

[4] 肖方样等,生物物理学报, 1992, 8: 318- 322 .

[5] 魏群等,生物化学杂志, 1993, 9:240-242.

[6] Tash, J. S. et al., J. Cell Biol., 1988, 106: 1625-1633.

[7] 孟样兵等,科学通报, 1993, 38(15): 1418-1421.

[8] Barry, K. et al., Brain Res., 1990, 511:21-29.

[9] Halpain, S. et al., Nature, 1990, 343:369-371.

[10] Halpain, S. et al., Nature, 1990, 343:237-239. 